\title{
Positronium formation in semicrystalline poly(ethylene terephthalate)
}

\author{
Li Xie and David W. Gidley \\ Department of Physics, University of Michigan, Ann Arbor, MI 48109, USA \\ and Hristo A. Hristov and Albert F. Yee* \\ Department of Materials Science and Engineering, University of Michigan, Ann Arbor, \\ MI 48109, USA \\ (Received 15 February 1993; revised 22 March 1993)
}

\begin{abstract}
The probability of positronium ( $\mathrm{Ps}$ ) formation in semicrystalline poly(ethylene terephthalate) (PET), has been investigated by means of positron annihilation lifetime spectroscopy (p.a.l.s.). The p.a.l.s. data are correlated with the structural characteristics of the samples, such as crystalline content, interlamellar spacing and lamella thickness. The results show an approximately linear decrease of the intensity $\left(I_{3}\right)$ of the long-lived orthopositronium (o-Ps) component with an increase in the crystalline content up to $53 \%$, while the lifetime remains practically constant. It seems that the other structural parameters do not affect the Ps formation. The decrease of $I_{3}$ cannot be completely accounted for if one assumes that $o$-Ps localizes in the amorphous phase only. It is suggested that the o-Ps also forms in the crystalline regions, due to the inhomogeneous electron density distribution along the $c$-axis of the PET lattice.
\end{abstract}

(Keywords: p.a.l.s.; semicrystalline PET; positronium formation)

\section{INTRODUCTION}

The successful application of positron annihilation lifetime spectroscopy (p.a.l.s.) as a quantitative method for studying the structural characteristics of polymeric materials depends on an understanding of the behaviour of the positrons injected into the substance. It has been found that in all known polymers the positron can exist as a free positron or form a bound state with the electrons from the medium, which is then known as positronium $(\mathrm{Ps})^{1-3}$. The typical lifetime of the positron is in the range $0.3-0.5 \mathrm{~ns}$, while the lifetime of the Ps depends on the spin state. The singlet state or parapositronium ( $p$-Ps) annihilates in $0.1-0.2 \mathrm{~ns}$, and investigations show that its lifetime $\left(\tau_{1}\right)$, as well as the $\mathrm{e}^{+}$lifetime $\left(\tau_{2}\right)$, are not sensitive to temperature and structural changes ${ }^{1-4}$. The triplet state or orthopositronium (o-Ps) has an intrinsic vacuum lifetime of $\sim 140 \mathrm{~ns}$, which in amorphous polymers is reduced to $\tau_{3} \sim 1.5-3 \mathrm{~ns}$, by 'pick-off' annihilation with an electron from the surrounding molecules. The fraction of the positrons forming $o-\operatorname{Ps}\left(I_{3}\right)$, and $\tau_{3}$ both depend on the temperature and structural changes in the host glass. It is widely accepted that $o$-Ps exhibits a strong tendency to localize in microvoids or small holes, where the electron density is lower than that in the bulk, and it has been found that $\tau_{3}$ increases with an increase in the hole volume ${ }^{2-4}$.

The positronium formation process in polymeric solids is not well understood. The most popular models (i.e. those due to Ore and Spur) use completely different approaches to explain the process of $o$-Ps formation and there is no clear evidence in favour of one or the other at the present time ${ }^{3}$. The experimental results show that

* To whom correspondence should be addressed

0032-3861/94/010014-04

(C) 1994 Butterworth-Heinemann Ltd.

POLYMER, 1994, Volume 35, Number 1 in porous resins and other porous materials $I_{3}$ is proportional to the total surface area of the pores ${ }^{3,5}$. It is assumed that in glassy polymers $I_{3}$ is correlated to the density of holes in the material, but the exact nature of this correlation, however, is not known ${ }^{6}$. In unoriented, semicrystalline polymers the picture is even more clouded, since one has to account for the many possible types of holes arising from the complex morphology of the spherulites commonly present in this class of materials. Based on results obtained from polyethylene with a crystallinity content higher than $50 \%$, a group of researchers ${ }^{1,2,7}$ has suggested that there are actually two long-lived $o$-Ps components, where the first $\left(\tau_{3} \sim 1 \mathrm{~ns}\right.$, $I_{3} \sim 10 \%$ ) is attributed to $o$-Ps annihilation in the chainfolded regions, and the second $\left(\tau_{4} \sim 2.4-2.6 \mathrm{~ns}\right.$, $I_{4} \sim 16-22 \%$ ) is attributed to 'pick-off' annihilation of $o$-Ps entrapped in the amorphous phase. Similar results have been reported for semicrystalline polytetrafluoroethylene and 1,4-polybutadiene ${ }^{8,9}$. Recent investigations of a series of poly(ether ether ketone) (PEEK) samples ${ }^{10}$ has led to the conclusion that there is only one long-lived component $\left(\tau_{3} \sim 1.8 \mathrm{~ns}\right)$, regardless of the presence or absence of the crystalline phase. It was also found that $I_{3}$ decreases linearly when the degree of crystallinity increases up to $30 \%$. On extrapolation to $100 \%$ crystallinity (i.e. a single crystal) $I_{3}$ decreases to zero, which has led to the conclusion that $o$-Ps formation is only possible in the amorphous phase of PEEK ${ }^{10}$. In the light of this somewhat ambiguous situation, the aim of this paper is to report a new experiment designed to shed further light on the subject. The data were obtained from a polymer with well-known structural and morphological parameters. The material used in this investigation, poly(ethylene terephthalate) (PET), has been known and 
Table 1 Thermal histories and structural parameters of the various PET samples

\begin{tabular}{lrrrrl}
\hline Sample & $\begin{array}{l}\text { Temp. } \\
\left({ }^{\circ} \mathrm{C}\right)\end{array}$ & $\begin{array}{l}\text { Time } \\
(\mathrm{h})\end{array}$ & \multicolumn{1}{l}{$\begin{array}{l}X \\
(\%)\end{array}$} & \multicolumn{1}{l}{$\begin{array}{l}\mathrm{l} \\
(\AA)\end{array}$} & \multicolumn{1}{l}{$\begin{array}{l}l \\
(\AA)\end{array}$} \\
\hline PET0 & & & 0.000 & & \\
PET1 & 95.000 & 0.500 & 0.300 & & \\
PET2 & 95.000 & 1.000 & 0.500 & & \\
PET3 & 95.000 & 1.500 & 8.300 & 92.000 & 30.000 \\
PET4 & 95.000 & 2.000 & 11.400 & 94.000 & 32.000 \\
PET5 & 95.000 & 3.000 & 16.700 & 93.000 & 31.000 \\
PET6 & 95.000 & 5.000 & 18.700 & 94.000 & 32.000 \\
PET7 & 95.000 & 12.000 & 19.800 & 93.000 & 33.000 \\
PET8 & 95.000 & 24.000 & 20.800 & 94.000 & 34.000 \\
PET9 & 95.000 & 48.000 & 22.900 & 95.000 & 34.000 \\
PET10 & 150.000 & 0.150 & 27.000 & 97.000 & 35.000 \\
PET11 & 150.000 & 2.000 & 30.200 & 98.000 & 36.000 \\
PET12 & 220.000 & 18.000 & 53.000 & 120.000 & 43.000 \\
PET13 & 245.000 & 24.000 & 47.700 & 210.000 & 83.000 \\
\hline
\end{tabular}

studied for several decades. The structures of the repeating chain units and the unit cells of PET and PEEK are quite different ${ }^{11}$. Regardless of the crystallization method the unit cell of PET is triclinic, and for quiescent crystallization the lattice parameters are as follows ${ }^{11}$ : $a=4.56 \AA, b=5.94 \AA, c=10.75 \AA, \alpha=98.5^{\circ}, \beta=118^{\circ}$, and $\gamma=112^{\circ}$. The melting temperature of PET $\left(\sim 255^{\circ} \mathrm{C}\right)$ is lower than that of PEEK $\left(335^{\circ} \mathrm{C}\right)$, while the densities of amorphous and crystalline PET are slightly higher than those of PEEK ${ }^{10,11}$. P.a.l.s. measurements, in combination with wide-angle X-ray diffraction (WAXD) and small-angle $X$-ray scattering (SAXS) measurements were performed on PET samples with crystalline contents ranging from 0 to $53 \%$. The temperature dependence of the p.a.l.s. response of a sample annealed at high temperature was also investigated.

\section{EXPERIMENTAL}

\section{Material}

The starting material is a commercial grade amorphous PET $\left(M_{\mathrm{w}}=50000\right.$ and $\left.M_{\mathrm{n}}=22000\right)$, prepared by injection moulding (Allied Signal Corporation). The samples were annealed at $95^{\circ} \mathrm{C}$ over increasing time periods (up to $48 \mathrm{~h}$, see Table I). Since at this temperature the crystalline content only reached $23 \%$, several other samples were prepared by thermal treatment at elevated temperatures in order to obtain higher degrees of crystallinity (Table I). After the annealing process, the specimens were surface ground to a thickness of $1.5 \mathrm{~mm}$ and then fine-polished.

\section{P.a.l.s.}

The p.a.l.s. measurements of the series of specimens were performed by using a conventional fast-timing coincidence method $^{12}$ in a temperature-controlled chamber at a constant temperature of $25 \pm 1^{\circ} \mathrm{C}$. The thermal response was investigated by heating the material up to $200^{\circ} \mathrm{C}$, in intervals of $25^{\circ} \mathrm{C}$, by a heater with a temperature control. The positron source $\left[\sim 1 \times 10^{4} \mathrm{~Bq}\right.$ $(\sim 30 \mu \mathrm{Ci})$ of $\left.{ }^{22} \mathrm{Na}\right]$ was deposited on the surface of an annealed single-crystal copper disc. As a result, $I_{3}$ measured herein is approximately half of that determined with the source sandwiched between two samples. The spectra were acquired and stored in a MicroVAX-based multichannel analyser. Each spectrum was acquired for $3 \mathrm{~h}$, and for some of the samples the measurement was repeated several times. The lifetime spectra were resolved into three or four components by using the PFPOSFIT program (for more details, see refs given in ref. 12).

\section{$W A X D$}

The WAXD data were collected by using a rotating anode Rigaku (RU 200BH) instrument with scintillation registration, in $\mathrm{CuK} \alpha$ radiation $(\lambda=1.54 \AA)$ and symmetrical transmission geometry. The diffraction curves, collected step by step over the angular interval $2 \theta=5-35^{\circ}$, were used to obtain the crystallinity index (relative crystallinity) of the PET specimens according to a procedure proposed by Statton ${ }^{13}$. Since the sample thickness was constant, the usual corrections of the experimental curves (i.e. background scattering and absorption) were not applied.

\section{$S A X S$}

The SAXS curves were obtained by using a diffractometer with Kratky-type collimation (Anton Paar, Austria), in FeK $\alpha$ radiation $(\lambda=1.94 \AA)$. The diffracted intensities were registered by a position-sensitive proportional counter (M-Brown, Germany) in $2 \theta$ intervals from $0.05-5^{\circ}$ simultaneously. The experimental curves were smoothed by Fourier filtering, de-smeared (infinitely long slit approximation ${ }^{14}$ ) and corrected for background and Lorenz polarization ${ }^{13}$. The corrected curves were used to obtain the long period $D$ by applying the Bragg formula $(D=\lambda /(2 \sin \theta))$, and the lamella thickness $l$ by using a cosine Fourier transformation of the curves ${ }^{13}$.

\section{Density}

The densities of samples 'PET12' and 'PET13' were measured at $23^{\circ} \mathrm{C}$ by the flotation method, using a carbon tetrachloride/n-heptane mixture. Since the densities of the amorphous and crystalline phases depend on the crystallization temperature $\left(T_{c}\right)^{15}$, the volume-fraction crystallinities were calculated from the experimental densities by using the following values: $\rho_{\mathrm{a}}=1.312 \mathrm{~g} \mathrm{~cm}^{-3}, \rho_{\mathrm{c}}=1.482 \mathrm{~g} \mathrm{~cm}^{-3}$ for sample 'PET12', and $\rho_{\mathrm{a}}=1.311 \mathrm{~g} \mathrm{~cm}^{-3}, \rho_{\mathrm{c}}=1.51 \mathrm{~g} \mathrm{~cm}^{-3}$ for sample 'PET13', where $\rho_{\mathrm{a}}$ and $\rho_{\mathrm{c}}$ are the densities of the amorphous and crystalline regions, respectively. The relative crystallinities derived from WAXD measurements were then converted into absolute values by using the crystallinities obtained from the two samples.

\section{RESULTS AND DISCUSSION}

In Table 1 the thermal histories of the various samples, together with the structural parameters derived from the WAXD, SAXS and density measurements, are presented. The experimental errors were estimated at $\pm 2 \AA$ for the long period and lamella thickness, and $\pm 1 \%$ for the degree of crystallinity. The results show that annealing at $95^{\circ} \mathrm{C}\left(T_{\mathrm{g}} \sim 72^{\circ} \mathrm{C}\right)$ increases the crystallinity content up to a level of $23 \%$, while the corresponding changes in the lamella thickness, and especially the long period, are very modest. Increasing the crystallization temperature gradually increases the crystallinity; a significant increase of the long period and the lamella thickness was measured at a $T_{\mathrm{c}}$ above $200^{\circ} \mathrm{C}$.

As discussed in the Introduction, the formation of $o$-Ps in both the crystalline and amorphous phases depends on the material under investigation. In order to clarify this 


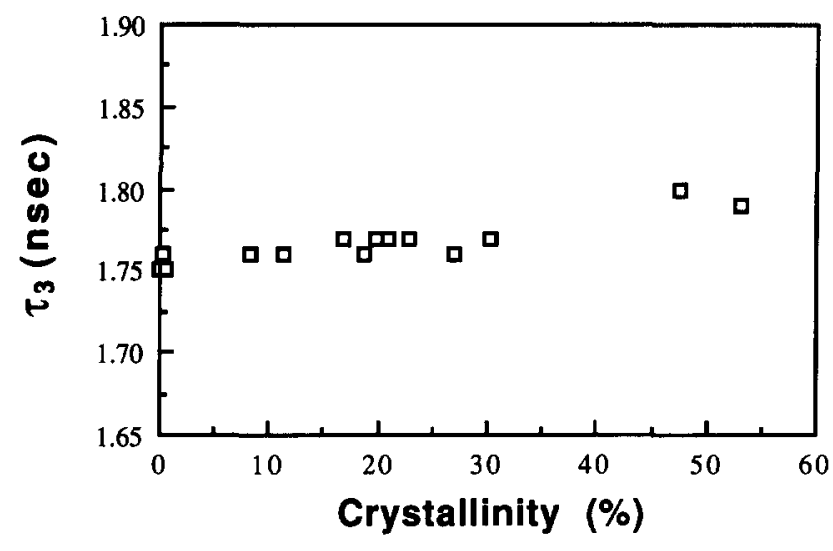

Figure 10 -Ps lifetime in semicrystalline PET as a function of the degree of crystallinity, where the rectangular plotting symbol corresponds to the experimental error along each axis

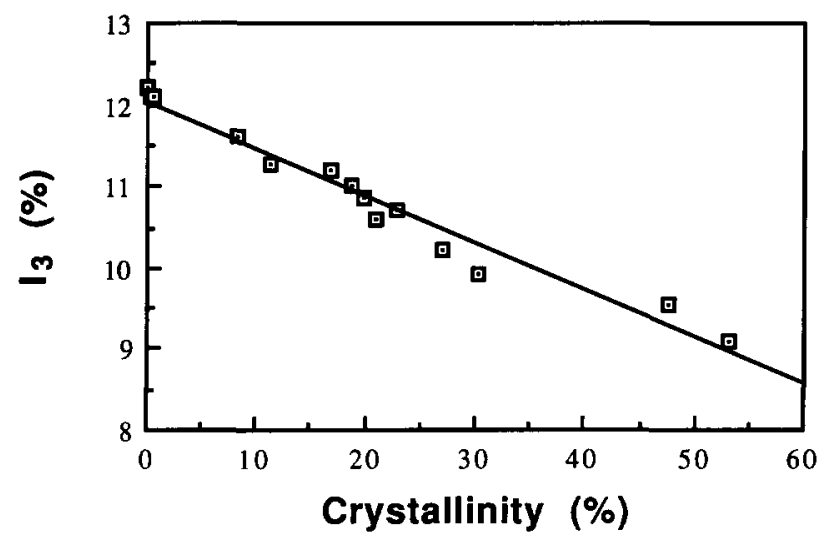

Figure $2 o$-Ps intensity change in semicrystalline PET as a function of the degree of crystallinity. The solid line is a least-squares fit to the experimental intensities, and the rectangular plotting symbol corresponds to the experimental error along each axis

question in the case of PET, we have collected 3-4 separate spectra for samples with crystallinities higher than $20 \%$. Each spectrum was fitted first with three and then with four exponential components. Since the four-component fitting requires some constraints ${ }^{7}$, we analysed the spectra by either fixing only the shortest lifetime ( $\tau_{1}$ in the range $0.1-0.2 \mathrm{~ns}$ ), or fixing both of the two short lifetimes $\left(\tau_{1}\right.$ in the range $\sim 0.1-0.2 \mathrm{~ns}, \tau_{2}$ in the range $0.3-0.5 \mathrm{~ns}$ ). Regardless of the constraints used, the four-component fit did not give consistent results: there was no significant improvement in $\chi^{2}$ and standard deviations were much higher than the respective values when the curves were fitted with three exponential components (unrestricted). The logical conclusions are that there is only one $o$-Ps component or if there are two components, then $\tau_{4}$ is too close to $\tau_{3}(\sim 1.77 \mathrm{~ns})$ or $\tau_{2}$ ( $\left.\sim 0.43 \mathrm{~ns} ; \tau_{1} \sim 0.15 \mathrm{~ns}\right)$ to be reliably resolved by the program.

Similar to the results previously reported for PEEK ${ }^{10}$, the $o$-Ps lifetimes, shown in Figure 1, are nearly constant with a slight rise in $\tau_{3}$ with increasing crystallinity. Taking into account that the density of the amorphous phase decreases with increasing crystallization temperature ${ }^{15}$, which should produce an increase in $\tau_{3}$, this result does not conflict with the assumption that $o$-Ps formation is possible in the amorphous phase only. If the above assumption is valid, one should expect a linear decrease of the $o$-Ps intensity with increasing crystallinity ${ }^{10}$. As shown in Figure 2, the $I_{3}$ decrease is reasonably linear when plotted as a function of the crystallinity, and moreover there is no correlation with the spherulite parameters, such as the long period and the lamella thickness. However, the extrapolation of the least-square fitted line gives $I_{3} \sim 6 \%$ at $100 \%$ crystallinity. This value, which is about half of the value of the $o$-Ps intensity of the initial material, strongly suggests that the crystalline phase contributes to the $o$-Ps formation. Therefore, one consistent interpretation of the data presented in Figures 1 and 2 is that the $o$-Ps lifetime and its corresponding intensity are $1.75 \mathrm{~ns}$ and $12 \%$, and $\sim 1.83 \mathrm{~ns}$ and $6 \%$, for the amorphous and crystalline phases, respectively. The two lifetimes are so close that a single $o$-Ps component with a weighted average lifetime and intensity is determined by the fitting.

There are several ways to understand the formation of $P s$ in the crystalline phase. It has been known ${ }^{3}$ that defects in crystals can serve as trapping sites for positrons. Annealing PET at temperatures close to the glass transition (samples annealed at $95^{\circ} \mathrm{C}$ ) undoubtedly produces highly defective crystals. On the other hand, an increase in the annealing time and/or $T_{c}$ considerably improves the crystalline quality ${ }^{11}$. If the structural defects were the main contributor to the Ps formation in the crystalline phase, then the $I_{3}$ decrease should be much steeper than that shown in Figure 2, and is most probably non-linear. This apparent inability of the defects in the crystalline phase to account for the observed trend in $I_{3}$ has led us to propose here an alternative site for $o$-Ps formation as a result of a special characteristic of the crystal structure of PET. In contrast to metals and smallmolecule crystals, polymers have never been observed to crystallize in close-packed structures. In addition, the morphology of the repeating chain unit very often imposes an inhomogeneous atomic/electronic density distribution within the unit cell. Figure 3 shows the chain conformation and the (100) plane of crystalline $\mathrm{PET}^{16}$. By using the lattice parameters, $\rho_{\mathrm{c}}$ and $\rho_{\mathrm{a}}$, one can compute that the electron density of the crystalline phase is $0.46 \mathrm{e} \AA^{-3}$, while the corresponding value for

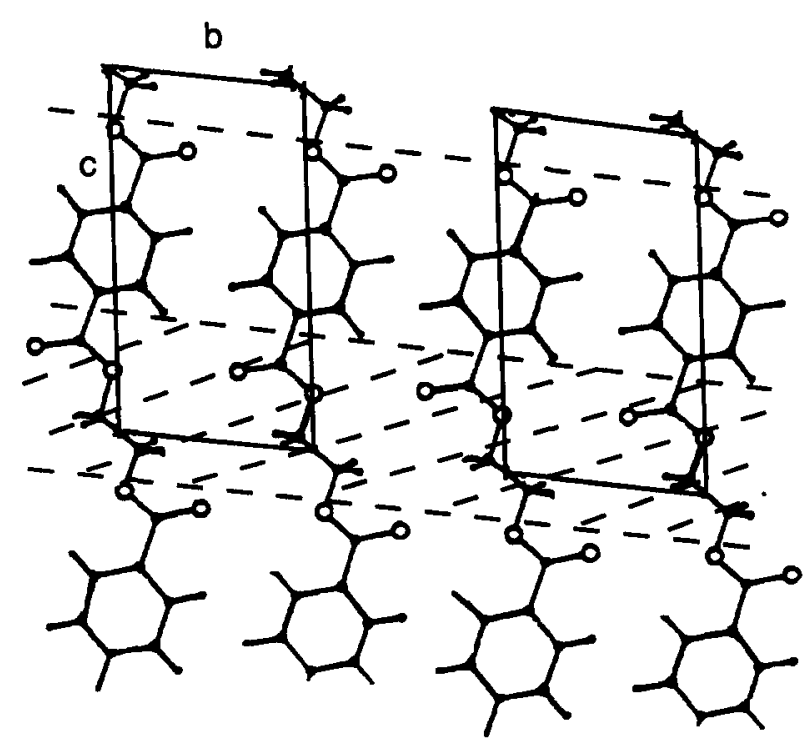

Figure 3 The (100) plane and the crystallographic unit cell of a PET crystal, where the shadowed portion represents an area with low electronic density (for details see text) 


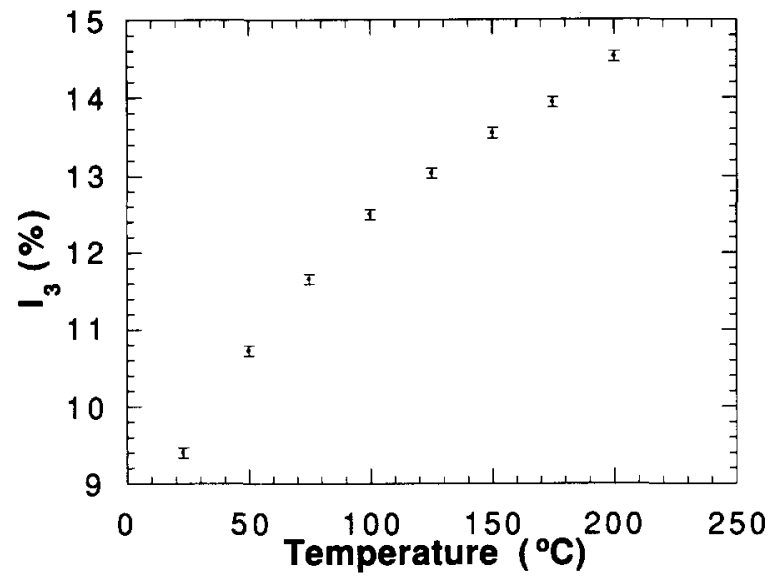

Figure 4 Temperature dependence of the $o$-Ps intensity of sample PET13 (annealed at $245^{\circ} \mathrm{C}$ )

the amorphous polymer is $0.41 \mathrm{e} \AA^{-3}$. If we now divide the crystal into two 'sublattices', as shown in Figure 3, the electron density of the shadowed portion will be only $0.37 \mathrm{e} \AA^{-3}$, which is lower than the average density of the amorphous phase. It should be noted that although the choice of the sublattices is not unique, the inhomogeneous electron distribution is a characteristic of the crystal structure of PET. It is conceivable that in the regions of reduced atomic/electron density even small lattice distortions, such as longrange thermal vibrations with frequencies $<10^{8} \mathrm{~Hz}$, can provide trapping sites for 0 -Ps formation. Such a bulk property could account for $o$-Ps formation in the crystalline phase and the respective $I_{3}$ dependence on crystallinity should be linear.

One avenue to explore the above hypothesis is to investigate the temperature dependence of the $o$-Ps intensity. In Figure 4 the $I_{3}$ temperature dependence for specimen PET13 (47\% crystallinity) is shown. Since the sample has been annealed for $24 \mathrm{~h}$ at $245^{\circ} \mathrm{C}$ it is safe to assume that no further annealing will occur during the course of these measurements. Our measurements of the thermal response of amorphous polycarbonate (PC) specimens (PC crystallization is extremely slow at all temperatures in the absence of a solvent) have shown that the $o$-Ps intensity levels off when the glass transition is approached $\left(T_{\mathrm{g}}=154^{\circ} \mathrm{C}\right)$, and remains effectively constant at higher temperatures. Similar results have been reported for amorphous polystyrene (PS) ${ }^{1}$ and an epoxy resin ${ }^{17}$. This phenomenon is most probably related to the fact that at temperatures close to the glass transition the concentration of 'holes' in the amorphous polymer reaches an equilibrium value, resulting in a constant probability for Ps formation ${ }^{17}$. The curve in Figure 4 demonstrates that $I_{3}$ increases continuously up to $200^{\circ} \mathrm{C}$. This result is consistent with our hypothesis that $o$-Ps may form in the PET crystal, and that the trapping sites are indeed thermally activated 'holes'.

Comparing the atomic structures of the PET and PEEK molecules ${ }^{11,18}$, we find that the distance between the benzene rings (along the $c$-axis) is $10.75 \AA$ vs. $5.08 \AA$. The benzene rings in the PEEK molecule are separated by a single atom only, which leads to a more homogeneous atomic/electronic density distribution along the $c$-axis. It is quite possible that in this case the intra-crystalline trapping sites for $o$-Ps formation are simply not present in the PEEK crystal.

\section{CONCLUSIONS}

A p.a.l.s. investigation of semicrystalline PET demonstrates that the probability of $o$-Ps formation decreases linearly with the increase of the crystalline content up to a level of $53 \%$. It seems that the other structural parameters (interlamellar spacing, lamella thickness and defect concentration) do not significantly affect the formation probability of $o$-Ps. It has been found that the decrease in $I_{3}$ with increasing crystallinity cannot be completely accounted for by assuming that $o$-Ps localizes only in the amorphous regions. It is suggested that $o$-Ps forms in the intra-crystalline regions of low atomic/electronic density, and that these sites appear to be thermally activated.

\section{ACKNOWLEDGEMENT}

This work was supported by the US Department of Energy under grant No. DE-FG02-88ER45366.

\section{REFERENCES}

1 Hamielec, A. E., Eldrup, M., Mogensen, O. and Jansen, P J. Macromol. Sci., Rev. Macromol. Chem. 1973, C9, 305

2 Stevens, J. R. in 'Methods in Experimental Physics', (Ed. R. A Fava), Vol. 16A, Academic Press, New York, 1980, Ch. 5, p. 371

3 Wang, S. J. and Jean, Y. C. in 'Positron and Positronium Chemistry' (Eds D. M. Shrader and Y. C. Jean), Elsevier, Amsterdam, 1988, Ch. 5, p. 255

4 Brandt, W. and Spirn, J. Phys. Rev. 1966, 142, 231

5 Venkateswaran, K., Cheng, K. L. and Jean, Y. C. J. Phys. Chem. $1984,88,2465$

6 Wang, Y. Y., Nakanishi, H. and Jean Y. C. J. Polym. Sci., Polym. Phys. Edn 1990, 28, 1431

7 Suzuki, T., Oki, Y., Numujiri, M., Miura, T., Kondo, K. and Ito, Y. J. Polym. Sci., Polym. Phys. Edn 1992, 30, 517

8 Brandt, W. and Winkenfeld, J. Phys. Rev. 1975, B12, 2579

9 Stevens, J. R. and Edwards, M. J. J. Polym. Sci., Polym. Lett. Edn 1970, 30, 297

10 Nakanishi, H., Jean, Y. C., Smith, E. G. and Sandreczki, T. C. J. Polym. Sci., Polym. Phys. Edn 1989, 27, 1419

11 Schultz, J. M. in 'Solid State Behavior of Linear Polyesters and Polyamides' (Eds J. M. Schultz and S. Fakirov), Prentice Hall, Englewood Cliffs, 1990, Ch. 2, p. 75

12 Liu, L. B., Gidley, D. and Yee, A. F. J. Polym. Sci., Polym. Phys. Edn 1992, 30, 231

13 Balta-Calleja, F. J. and Vonk, C. G. in 'X-Ray Scattering of Synthetic Polymers' (Ed. A. D. Jenkins), Elsevier, Amsterdam, 1989 , p. 178

14 Glatter, O. in 'Small Angle X-Ray Scattering' (Eds O. Glatter and O. Kratky), Academic Press, New York, 1982, p. 119

15 Bornschlegl, E. and Bonart, R. Colloid Polym. Sci. 1980, 258, 319

16 Daubeny, R., Bunn, C. W. and Brown, J. C. Proc. R. Soc. London, A 1954, 226, 531

17 Nakanishi, H., Wang, Y. Y., Jean, Y. C., Sandreczki, T. C. and Ames, D. P. in 'Positron Annihilation in Fluids' (Ed. S. C. Sharma), World Scientific, Singapore, 1988, p. 781

18 Wakelin, N. T. Polym. Commun. 1984, 25, 306 